\title{
Depth-predicting score for differentiated early gastric cancer
}

\author{
Seiichiro Abe $\cdot$ Ichiro Oda $\cdot$ Taichi Shimazu • \\ Tetsu Kinjo • Kazuhiro Tada • Taku Sakamoto • \\ Chika Kusano $\cdot$ Takuji Gotoda
}

Received: 31 March 2010/Accepted: 24 August 2010/Published online: 17 February 2011

(C) The International Gastric Cancer Association and The Japanese Gastric Cancer Association 2011

\begin{abstract}
Background Intramucosal and minute submucosal (M-SM1; $<500 \mu \mathrm{m}$ in depth) differentiated gastric cancers, which have a negligible risk of lymph node metastasis, are the targets for endoscopic resection. However, there have been few reports about the endoscopic distinction between these cancers and cancers with deeper submucosal invasion (SM2; $\geq 500 \mu \mathrm{m}$ in depth). The aim of this retrospective study was to analyze the differences in the endoscopic features between M-SM1 and SM2 cancers, and to develop a simple scoring model to predict the depth of these early gastric cancers.

Methods We analyzed 853 differentiated early gastric cancers treated endoscopically or surgically as a derivation group. Endoscopic images were reviewed to determine the relationship between depth of invasion and the following endoscopic features: tumor location, macroscopic type, tumor size, and endoscopic findings (remarkable redness, uneven surface, margin elevation, ulceration, and enlarged folds). Secondly, we created a depth-predicting model based on the obtained data and applied the model to 211 validation samples.
\end{abstract}

S. Abe · I. Oda $(\bowtie) \cdot$ T. Kinjo $\cdot$ K. Tada $\cdot$ T. Sakamoto .

C. Kusano - T. Gotoda

Endoscopy Division, National Cancer Center Hospital,

5-1-1 Tsukiji, Chuo-ku, Tokyo 104-0045, Japan

e-mail: ioda@ncc.go.jp

T. Shimazu

Epidemiology and Prevention Division,

Research Center for Cancer Prevention and Screening,

National Cancer Center, Tokyo, Japan

C. Kusano $\cdot$ T. Gotoda

Department of Gastroenterology and Hepatology,

National Center for Global Health and Medicine, Tokyo, Japan
Results On logistic regression analysis, tumor size more than $30 \mathrm{~mm}$, remarkable redness, uneven surface, and margin elevation were significantly associated with deeper submucosal cancers. A depth-predicting score was created by assigning 2 points for margin elevation and tumor size more than $30 \mathrm{~mm}$, and 1 point for each of the other endoscopic features. When validation lesions of 3 points or more were diagnosed as deeper submucosal cancers, the sensitivity, specificity, and accuracy as evaluated by three endoscopists were $29.7-45.9,93.1-93.7$, and 82.5-84.8\%, respectively. Conclusions The depth-predicting score could be useful in the decisions on treatment strategy for differentiated M-SM1 early gastric cancers.

Keywords Early gastric cancer - Depth - Diagnosis · Endoscopy

\section{Introduction}

Endoscopic resection in patients with early gastric cancer (EGC) is less invasive and more economical than conventional surgery. The negligible incidence of lymph node metastasis in certain stages of EGC means that, in selected cases, patients can be cured with such therapies. Gotoda et al. [1] concluded that among 5265 patients who underwent gastrectomy, there was no lymph node involvement in differentiated mucosal (M) gastric cancers without lymphatic or vessel invasion when the cancers were smaller than $3 \mathrm{~cm}$ in diameter with ulceration, or any size without ulceration. Differentiated minute submucosal (SM1, $<500 \mu \mathrm{m}$ in depth) cancers without lymphatic or venous involvement and cancers smaller than $3 \mathrm{~cm}$ also showed no lymph node involvement [1]. The endoscopic submucosal dissection (ESD) technique using an insulation-tipped 
diathermic knife or other endo-knives could technically achieve one-piece resection for such lesions [2-7]. It is important to distinguish M-SM1 cancers from deeper submucosal (SM2; $\geq 500 \mu \mathrm{m}$ in depth) cancers, which have the possibility of lymph node metastasis, for making the proper decision on treatment strategy.

Thus, preoperative determination of the depth of invasion is important. Although the usefulness of endoscopic ultrasonography (EUS) has been reported, with this modality it is impossible to distinguish M-SM1 from SM2 definitively $[8,9]$. Conventional endoscopy is the initial route of EGC detection, but there have been few reports comparing the endoscopic features of EGC stages M-SM1 and SM2. Furthermore, no objective criteria regarding the depth of invasion exist, and many endoscopists diagnose based on their own experiences. The aim of this retrospective study was to analyze the differences in the endoscopic features between M-SM1 and SM2, and to develop a simple model to predict the depth of these EGCs.

\section{Materials and methods}

Analyzed lesions and review methods

A total of 880 consecutive differentiated EGCs were treated endoscopically or surgically between 2001 and 2003 at the National Cancer Center Hospital in Tokyo. Twentyseven lesions were excluded because precise endoscopic findings could not be depicted [eight detected in remnant stomach, six after esophagectomy, six local recurrences after endoscopic mucosal resection (EMR), five with insufficient endoscopic images, one with a tattoo, and another with an endo-clip artifact].

The remaining 853 differentiated EGCs (M 592, SM1 111, SM2 150, mean patient age of 65.6 years, 686 male and 167 female patients) were analyzed as a derivation group. An endoscopist (S.A.), experienced with more than 5000 gastroscopies, reviewed conventional endoscopic images without histological information about depth. The following characteristics were evaluated: tumor location (upper, middle, and lower), tumor size (mm), macroscopic type, and five other endoscopic findings that are widely accepted as markers of deeper submucosal invasion among Japanese endoscopists, with some minor variations (remarkable redness, uneven surface, margin elevation, ulceration, and enlarged folds) [10, 11].

Subsequently, we made a simple and practical scoring model (depth-predicting score, DPS) to distinguish M-SM1 from SM2 cancers, based on the analyzed data in the derivation group. Three endoscopists (S.A., T.K., and K.T., each experienced with more than 5000 gastroscopies) evaluated the endoscopic findings and investigated the sensitivity, specificity, and accuracy of our DPS in our validation set, consisting of 211 differentiated EGCs treated between January and June in 2000 at our hospital.

Conventional white-light endoscopy (video-endoscope Q240 or Q260; Olympus Medical Systems, Tokyo, Japan) was used for pretreatment endoscopic examination. In addition, surface details were enhanced by indigo-carmine chromoendoscopy.

\section{Definitions}

The EGC macroscopic and histological types in the enrolled patients were decided according to the Japanese classification of gastric carcinoma [12]. We divided the macroscopic types into three groups: IIa (elevated lesions such as $0 \mathrm{I}, 0 \mathrm{IIa}$, and $0 \mathrm{I}+\mathrm{IIa}$ ), IIc (depressed lesions such as $0 \mathrm{IIc}, 0 \mathrm{IIc}+\mathrm{III}$, and 0 III + IIc), and IIa + IIc (combined type, such as 0 IIa + IIc and 0 IIc + IIa). Histological type was diagnosed based on the predominant tumor pattern and then divided into two types; differentiated type and undifferentiated type. Well differentiated, moderately differentiated, and papillary adenocarcinoma were defined as differentiated type.

We described five endoscopic features in this study. Remarkable redness was defined as a reddish area similar to regenerative epithelium (Fig. 1). Nodulations in the tumor's surface were considered an uneven surface (Fig. 2). Margin elevation referred to the finding of a protruding edge surrounding the tumors, including submucosal tumor like component with a limited amount of air insufflation (Fig. 3a, b). Either a scar or an ulcerative area within the tumors was evaluated as ulceration (Fig. 4). Finally, enlarged folds included any thickened or merged convergent folds (Fig. 5).

\section{Statistical methods}

To identify the variables that were significantly more common in SM2, the endoscopic data were initially

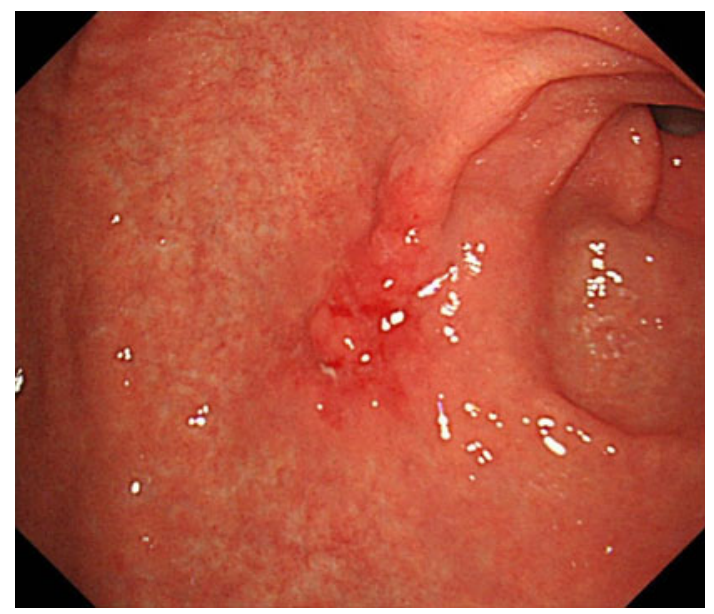

Fig. 1 Remarkable redness: endoscopic picture shows unusual redness inside the lesion 


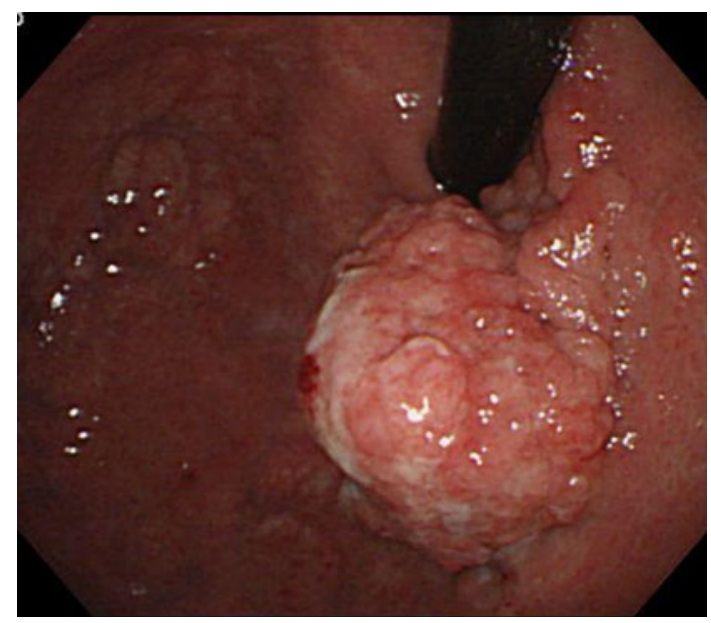

Fig. 2 Uneven surface: nodular mucosa can be seen
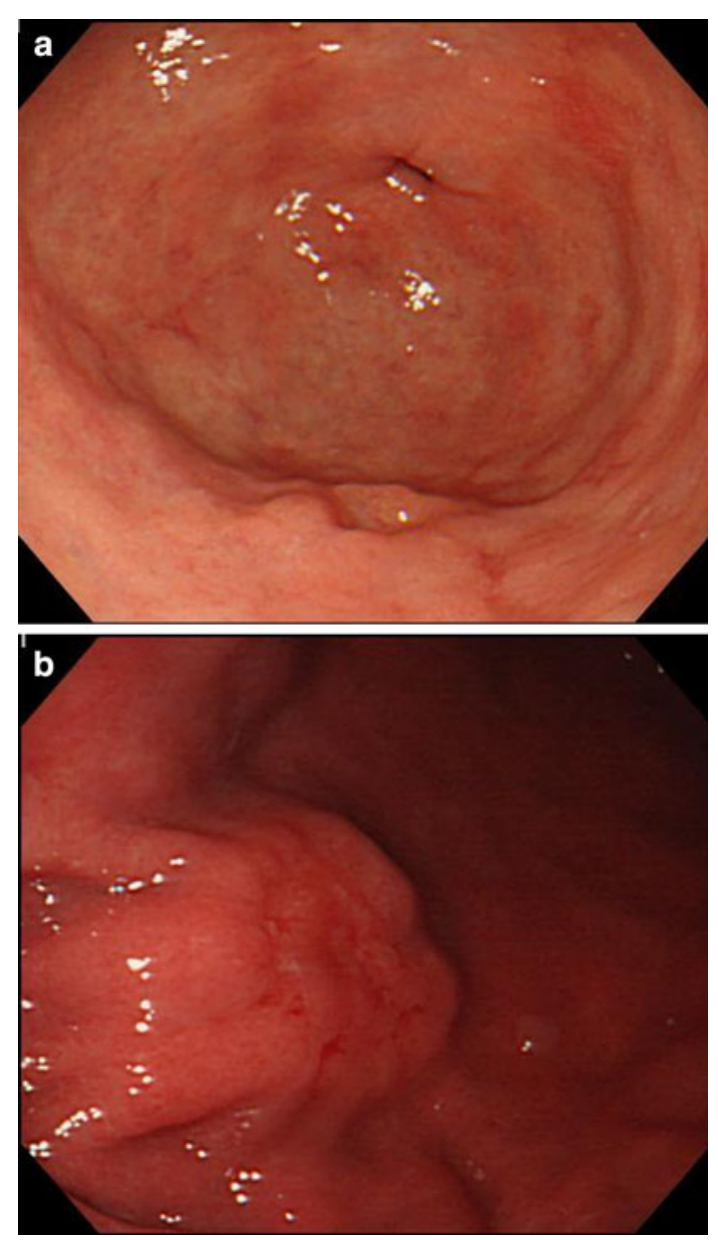

Fig. 3 a Margin elevation: endoscopic picture of surrounding elevation. b Margin elevation: endoscopic picture of submucosal tumor like component can be demonstrated from the view with a limited amount of air insufflation

evaluated with Student's $t$ test for tumor size and the $\chi^{2}$ test for other endoscopic features. We then entered the candidate variables into a logistic regression analysis.

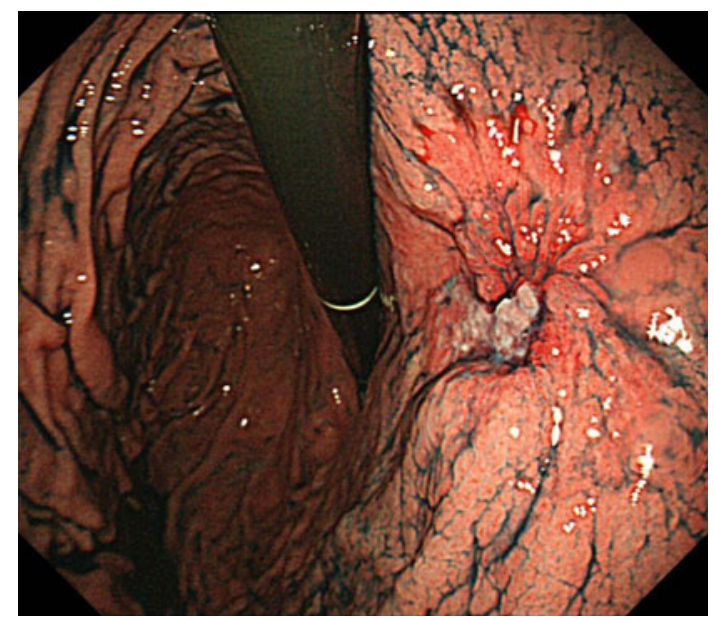

Fig. 4 Ulceration: endoscopic picture of ulceration

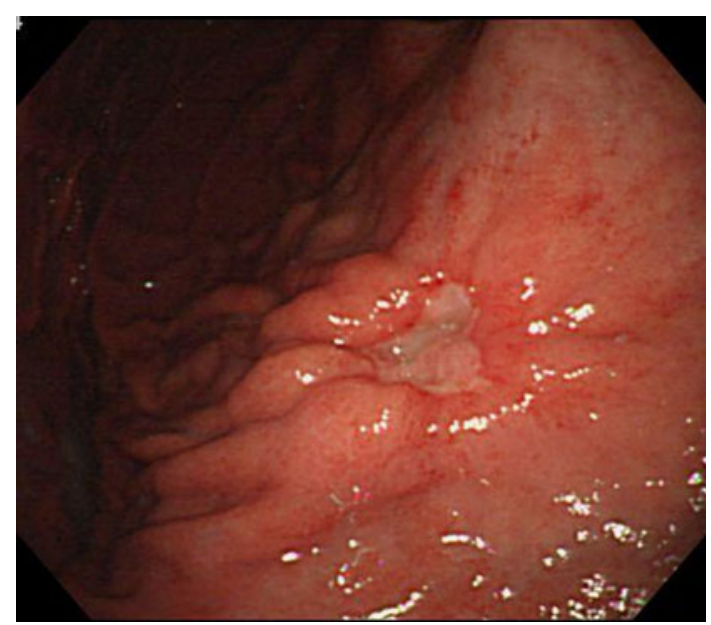

Fig. 5 Enlarged folds: thickened or merged folds can be seen toward the inside of the lesion

Endoscopic features independently and statistically associated with SM2 penetration were selected as examination items for the DPS. The relative weighting of each DPS variable was based on its $\beta$-coefficient in the logistic regression analysis. The significance level was set at $5 \%$ for each analysis. A $p$ value of $<0.05$ was considered significant.

\section{Results}

Analysis of endoscopic features

Table 1 shows the histological and therapeutic characteristics of both the derivation and validation groups. There were no significant differences between the two groups in the depth of invasion, histological type, or treatment strategies. 
Table 1 Histological and therapeutic characteristics

\begin{tabular}{llll}
\hline & $\begin{array}{l}\text { Derivation } \\
\text { group } \\
(n=853)\end{array}$ & $\begin{array}{l}\text { Validation } \\
\text { group } \\
(n=211)\end{array}$ & $p$ value \\
\hline $\begin{array}{l}\text { Depth (M-SM1/SM2) } \\
\text { Histological type }\end{array}$ & $703 / 150$ & $175 / 36$ & NS* $^{*}$ \\
Well & 732 & 185 & NS* \\
$\begin{array}{l}\text { Moderately } \\
\text { Papillary }\end{array}$ & 109 & 25 & \\
Treatment & 12 & 1 & \\
EMR/ESD & & & NS* \\
Surgery & 632 & 171 & \\
\hline
\end{tabular}

$M$-SM1 intramucosal and minute submucosal $(<500 \mu \mathrm{m}$ in depth) cancers, SM2 deeper submucosal ( $\geq 500 \mu \mathrm{m}$ in depth) cancers, well well-differentiated adenocarcinoma, moderately moderately differentiated adenocarcinoma, papillary papillary adenocarcinoma, EMR, endoscopic mucosal resection, ESD endoscopic submucosal dissection, NS not significant

* $\chi^{2}$ test

In the derivation group, there was no significant difference in tumor location between M-SM1 and SM2. SM2 gastric cancers were significantly larger and were characterized as IIa + IIc. According to the endoscopic features, we also found statistically significant differences in remarkable redness, uneven surface, margin elevation, ulceration, and enlarged folds (Table 2).

The tumor size cutoff was set at $30 \mathrm{~mm}$ with a cross point between the receiver operating characteristic (ROC) curve against SM2 and the $45^{\circ}$ line, which represented the ROC curve of a test whose decision ability is no better than chance (Fig. 6). Tumor size more than $30 \mathrm{~mm}$ was determined as a variable in multivariate analysis.

In the logistic regression analysis, tumor size (more than $30 \mathrm{~mm}$ ), macroscopic type, and endoscopic features which were significantly more common in SM2 by univariate analysis were investigated. As a result, margin elevation, tumor size (more than $30 \mathrm{~mm}$ ), remarkable redness, and uneven surface were significantly associated with SM2 EGCs (Table 3).

\section{Establishment of depth-predicting score}

The DPS was created based on the above results. One point was given for remarkable redness and uneven surface, while margin elevation and tumors more than $30 \mathrm{~mm}$ were scored with 2 points because the relative magnitude of the $\beta$-coefficient was roughly twice that of other variables. Thus, the range of the resulting DPS was 0-6 points (Table 4). A total of 3 points was defined as the cutoff between M-SM1 and SM2. This was done in order to balance the power for SM2 selection and minimize the
Table 2 Endoscopic comparison between M-SM1 and SM2 in derivation group

\begin{tabular}{llll}
\hline & $\begin{array}{l}\text { M-SM1 } \\
(n=703)\end{array}$ & $\begin{array}{l}\text { SM2 } \\
(n=150)\end{array}$ & $p$ value \\
\hline Location & & & \\
U & 134 & 38 & NS* \\
M & 257 & 35 & \\
L & 312 & 77 & \\
Tumor size (mm) & & & \\
Mean, range & $19.2(3-120)$ & $31.6(5-120)$ & $<0.0001^{*} *$ \\
Macroscopic type & & & \\
IIa & 178 & 30 & $<0.0001^{*}$ \\
IIc & 458 & 88 & \\
IIa + IIc & 67 & 32 & $<0.0001^{*}$ \\
Endoscopic features & & & $<0.0001^{*}$ \\
Remarkable redness & $160(22.8 \%)$ & $70(46.7 \%)$ & $<0.0001^{*}$ \\
Uneven surface & $72(10.2 \%)$ & $47(31.3 \%)$ & $<0.0001^{*}$ \\
Margin elevation & $110(15.6 \%)$ & $82(54.7 \%)$ & \\
Ulceration & $152(21.6 \%)$ & $57(38.0 \%)$ & $<11(7.3 \%)$ \\
Enlarged folds & $7(1.0 \%)$ & $11 \%$ & $<0.001^{*}$ \\
\hline
\end{tabular}

$U$ upper, $M$ middle, $L$ lower

$* \chi^{2}$ test, ** Student's $t$ test

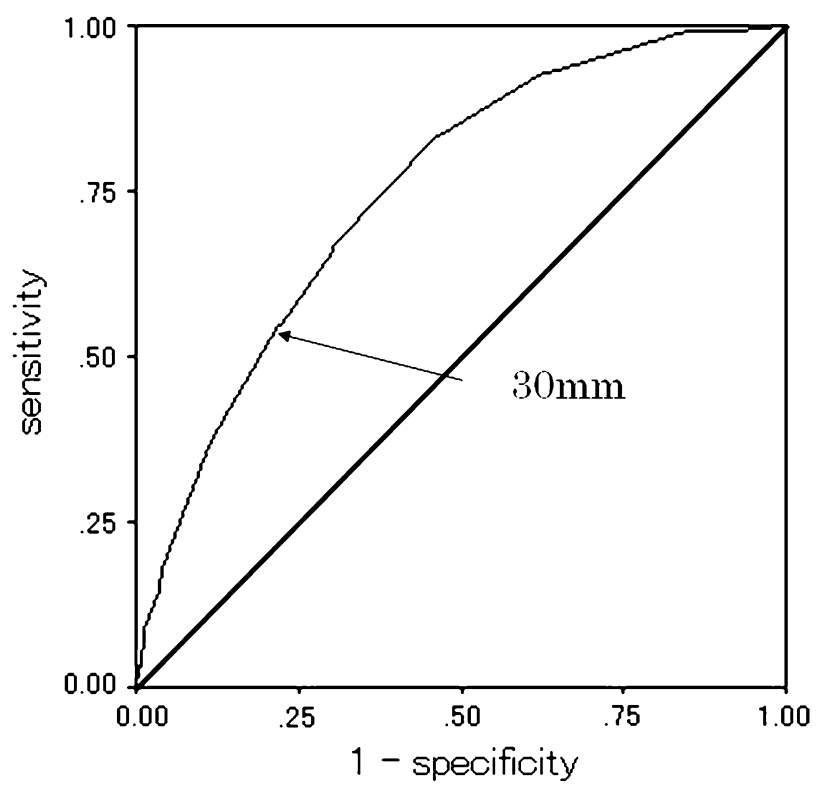

Fig. 6 Receiver operating characteristic curve for tumor size and the sensitivity of submucosal cancers $\geq 500 \mu \mathrm{m}$ in depth (SM2): the arrow (30-mm diameter) shows the cutoff point between intramucosal and minute submucosal $<500 \mu \mathrm{m}$ in depth (M-SM1) and SM2 cancers

population for overtreatment. The sensitivity, specificity, and accuracy of the proposed DPS were $57.3 \%$ (95\% confidence interval [CI] 49.4-65.3\%), 86.2\% (95\% CI 
Table 3 Multivariate logistic regression analysis

\begin{tabular}{|c|c|c|c|}
\hline & $\beta$-coefficient & $\begin{array}{l}\text { Odds ratio } \\
(95 \% \mathrm{CI})\end{array}$ & $p$ value \\
\hline Margin elevation & 7.838 & $6.221(3.938-9.825)$ & $<0.0001$ \\
\hline $\begin{array}{l}\text { Tumor size (more } \\
\text { than } 30 \mathrm{~mm} \text { ) }\end{array}$ & 6.570 & $4.937(3.066-7.951)$ & $<0.0001$ \\
\hline Remarkable redness & 3.411 & $2.087(1.367-3.186)$ & 0.0006 \\
\hline Uneven surface & 3.343 & $2.306(1.413-3.764)$ & 0.0008 \\
\hline
\end{tabular}

CI confidence interval

Evaluated items in multiple logistic regression analysis were followed: tumor size more than $30 \mathrm{~mm}$, macroscopic type (IIa + IIc), remarkable redness, uneven surface margin elevation, ulceration and enlarged folds. Only the statistically significant items are listed in the table

Table 4 Proposed depth-predicting score

\begin{tabular}{lll}
\hline Factor & Points & \\
\cline { 2 - 3 } & Present & Absent \\
\hline Margin elevation & 2 & 0 \\
Tumor size (more than $30 \mathrm{~mm})$ & 2 & 0 \\
Remarkable redness & 1 & 0 \\
Uneven surface & 1 & 0 \\
\hline
\end{tabular}

$83.7-88.8 \%$ ), and $81.1 \%$ (95\% CI $78.5-83.8 \%$ ), respectively (Fig. 7).

Finally, we applied the suggested DPS model to the 211 validation lesions without any histological information. When we considered 3 points or more as SM2, the sensitivity, specificity, and accuracy of the proposed DPS, assigned by the three endoscopists, were 29.7-45.9, 93.1-93.7, and 82.5-84.8\%, respectively. When we divided the validation group into "IIa" and "IIc/IIa + IIc", the sensitivity, specificity, and accuracy were 50.0-83.3, 92.6-96.3, and $91.7 \%$ (by all three endoscopists) for IIa lesions and 25.8-38.7, 92.5-93.3, and $78.8-82.1 \%$ for IIc/IIa + IIc lesions (Table 5).

\section{Discussion}

Patients' quality of life is one of the most important issues in EGC treatment, because the prognosis of EGC is favorable [13]. Differentiating endoscopically resectable M-SM1 gastric cancers from surgically resectable SM2 lesions is of great significance, given the low risk of lymph node metastases with the former. In conventional endoscopic diagnosis for these EGCs, however, endoscopists have had to empirically estimate the depth of invasion, as no objective criteria existed.

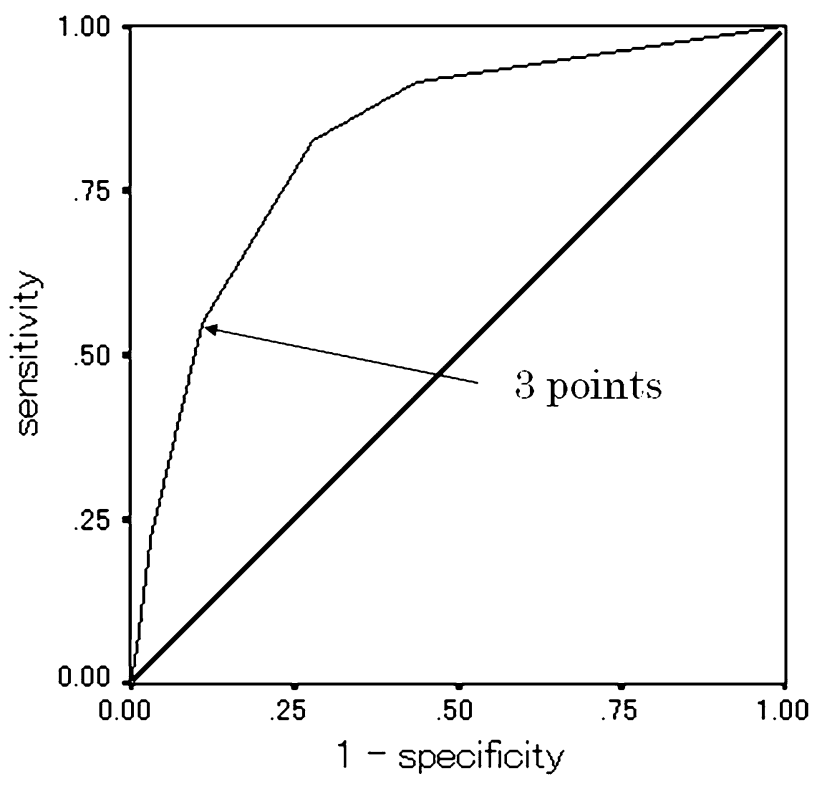

Fig. 7 Receiver operating characteristic curve for depth-predicting score (DPS) and the sensitivity of SM2: the arrow (3 points) shows the cutoff point between M-SM1 and SM2

Table 5 Diagnostic sensitivity and specificity by depth-predicting score according to macroscopic type in the validation group

\begin{tabular}{llll}
\hline \multicolumn{2}{c}{ IIa } & IIc/IIa + IIc & Total \\
\hline Endoscopist 1 & & & \\
Sensitivity & $50.0 \%(3 / 6)$ & $25.8 \%(8 / 31)$ & $29.7 \%(11 / 37)$ \\
Specificity & $96.3 \%(52 / 54)$ & $92.5 \%(111 / 120)$ & $93.7 \%(163 / 174)$ \\
Accuracy & $91.7 \%(55 / 60)$ & $78.8 \%(119 / 151)$ & $82.5 \%(174 / 211)$ \\
Endoscopist 2 & & \\
Sensitivity & $83.3 \%(5 / 6)$ & $38.7 \%(12 / 31)$ & $45.9 \%(17 / 37)$ \\
Specificity & $92.6 \%(50 / 54)$ & $93.3 \%(112 / 120)$ & $93.1 \%(162 / 174)$ \\
Accuracy & $91.7 \%(55 / 60)$ & $82.1 \%(124 / 151)$ & $84.8 \%(179 / 211)$ \\
Endoscopist 3 & & & \\
Sensitivity & $50.0 \%(3 / 6)$ & $35.8 \%(11 / 31)$ & $37.8 \%(14 / 37)$ \\
Specificity & $96.3 \%(52 / 54)$ & $92.5 \%(111 / 120)$ & $93.7 \%(163 / 174)$ \\
Accuracy & $91.7 \%(55 / 60)$ & $80.8 \%(122 / 151)$ & $83.9 \%(177 / 211)$ \\
\hline
\end{tabular}

The first aim of this retrospective study was to analyze the differences in conventional endoscopic features between M-SM1 and SM2 EGCs. We found that tumor size more than $30 \mathrm{~mm}$, margin elevation, uneven surface, and remarkable redness were significantly associated with an increased risk of SM2 invasion according to logistic regression analysis.

There have been few reports about the usefulness of conventional endoscopy for predicting depth of invasion. The overall accuracy rates for determining depth of invasion of EGCs were between 63 and $73 \%$ by non-objective criteria [11, 14, 15]. Namieno et al. [16] concluded that 
macroscopic appearance, histological differentiation, and tumor size were associated with submucosal invasion. However, they did not analyze the morphologic features of the tumors.

Although we used endoscopy in the present study, EUS can also show the depth of invasion clearly. The introduction of high-frequency thin probes has allowed target scanning with high resolution under endoscopic control [8, 9]. In spite of some excellent accuracy data [17], there have been no significant differences between EUS and endoscopy in terms of depth accuracy [14].

Considering the need for simple and objective diagnosis, we proposed an endoscopic determination for the depth of invasion of differentiated EGCs by the DPS described here, based on our analysis of the derivation group. The DPS could be used to determine an appropriate treatment strategy for the validation group with 82.5-84.8\% accuracy. Based on macroscopic type, the accuracy for elevated lesions tended to be better than that for the depressed and combined lesions.

Although specificity was good in steering M-SM1 cancers toward endoscopic treatment, low sensitivity was a weak point of the DPS. Selected endoscopic features may not reflect microscopic SM2 invasion. Also, each variable was considered as only either present or absent. If the significance of each finding had been taken into consideration, the sensitivity and accuracy of the score may have increased. However, this would have complicated the DPS, and was therefore not done.

EUS could be omitted for lesions with a DPS of less than 2 points and endoscopic resection performed, except for large ulcerative lesions more than $30 \mathrm{~mm}$ in diameter. Lesions with a DPS of 3 points or more may be considered as candidates for additional EUS, potentially providing more precise prediction. By using this simple diagnostic model, appropriate treatment strategies can be determined for differentiated M-SM1 EGCs, while saving time and cost as compared to EUS being done for all cases.

The limitation of this investigation was the retrospective design at a single institution. Further research in a prospective study is needed to investigate the utility of the DPS in combination with EUS for lesions with a DPS of 3 points or greater.

In conclusion, the proposed DPS may be useful in making treatment decisions for differentiated M-SM1 EGCs.

Acknowledgments The authors had the generous support and encouragement of Dr. Hitoshi Kondo (The Center for Digestive Diseases, Tonan Hospital, Japan). Also, we wish to thank Dr. Jeffery Paul Gaboury (Department of Surgery, University of Saskatchewan, Canada) and Dr. Satchan Takaya (Division of Infectious Diseases, University of Saskatchewan, Canada) for correcting the English used in this manuscript.

\section{References}

1. Gotoda T, Yanagisawa A, Sasako M, Ono H, Nakanishi Y, Shimoda $\mathrm{T}$, et al. Incidence of lymph node metastasis from early gastric cancer: estimation with a large number of cases at two large centers. Gastric Cancer. 2000;3:219-25.

2. Ono H, Kondo H, Gotoda T, Shirao K, Yamaguchi H, Saito D, et al. Endoscopic mucosal resection for treatment of early gastric cancer. Gut. 2001;48:225-9.

3. Oyama T, Kikuchi Y. Aggressive endoscopic mucosal resection in the upper GI tract. Hook knife EMR method. Minim Invasive Ther Allied Technol. 2002;11:291-5.

4. Yahagi N, Fujishiro M, Kakushima N, Kobayashi K, Hashimoto T, Oka M, et al. Endoscopic submucosal dissection for early gastric cancer using the tip of an electrosurgical snare (thin type). Dig Endosc. 2004;16:34-8.

5. Yamamoto H, Kawata H, Sunada K, Sasaki A, Nakazawa K, Miyata T, et al. Successful en-bloc resection of large superficial tumors in the stomach and colon using sodium hyaluronate and small-caliber-tip transparent hood. Endoscopy. 2003;35:690-4.

6. Ono H, Hasuike N, Inui T, Takizawa K, Ikehara H, Yamaguchi $\mathrm{Y}$, et al. Usefulness of a novel electrosurgical knife, the insulation-tipped diathermic knife-2, for endoscopic submucosal dissection of early gastric cancer. Gastric Cancer. 2008;1:47-52.

7. Oda I, Saito D, Tada M, Iishi H, Tanabe S, Oyama T, et al. A multicenter retrospective study of endoscopic resection for early gastric cancer. Gastric Cancer. 2006;9:262-70.

8. Ohashi S, Segawa K, Okamura S, Mitake M, Urano H, Shimodaira M, et al. The utility of endoscopic ultrasonography and endoscopy in the endoscopic mucosal resection of early gastric cancer. Gut. 1999;45:599-604.

9. Ichikawa T, Kudo M, Matsui S, Okada M, Kitano M. Endoscopic ultrasonography with three miniature probes of different frequency is an accurate diagnostic tool for endoscopic submucosal dissection. Hepatogastroenterology. 2007;54:325-8.

10. Ono H, Yoshida Y. Endoscopic diagnosis of the depth of cancer invasion for gastric cancer. Stomach Intest. 2001;36:334-40. (in Japanese with English abstract).

11. Sano T, Okuyama Y, Kobori O, Shimizu T, Morioka Y. Early gastric cancer endoscopic diagnosis of depth of invasion. Dig Dis Sci. 1990;35:1340-4.

12. Japanese Gastric Cancer Association. Japanese classification of gastric carcinoma, 2nd English edition. Gastric Cancer. 1998; $1: 10-24$.

13. Sasako M, Kinoshita T, Maruyama K. Prognosis of early gastric cancer. Stomach Intest. 1993;28(Suppl):139-46. (in Japanese with English abstract).

14. Yanai H, Noguchi T, Mizumachi S, Tokiyama H, Nakamura H, Tada M, et al. A blind comparison of the effectiveness of endoscopic ultrasonography and endoscopy in staging early gastric cancer. Gut. 1999;44:361-5.

15. Shirao K, Saito D, Yamaguchi H, Shirato M, Fukuda H, Yoshida S. Accuracy of preoperative diagnosis of early gastric cancer on submucosal invasion: a study for indication of endoscopic treatment. Stomach Intest. 1992;27:1175-83. (in Japanese with English abstract).

16. Namieno T, Koito K, Hiigashi T, Takahashi M, Shimamura T, Yamashita K, et al. Endoscopic prediction of tumor depth of gastric carcinoma for assessing the indication of its limited resection. Oncol Rep. 2000;7:57-61.

17. Yoshida S, Tanaka S, Kunihiro K, Mitsuoka Y, Hara M, Kitadai Y. Diagnostic ability of high-frequency ultrasound probe sonography in staging early gastric cancer, especially for submucosal invasion. Abdom Imaging. 2005;30:518-23. 